\title{
Kinetic Modelling of Atmospheric Pressure Nitrogen Plasma
}

\author{
Md. Ziaur Rahman ${ }^{1}$, Mohammed Mynuddin ${ }^{2}$ \\ ${ }^{1}$ Department of Civil Engineering, Dhaka International University, Dhaka, Bangladesh \\ ${ }^{2}$ Department of Electrical \& Electronics Engineering, Georgia Southern University, Statesboro, USA
}

Email address:

ziaapeeru@gmail.com (Md. Z. Rahman), myn101eee@gmail.com (M. Mynuddin)

\section{To cite this article:}

Md. Ziaur Rahman, Mohammed Mynuddin. Kinetic Modelling of Atmospheric Pressure Nitrogen Plasma. American Journal of Modern Physics. Vol. 7, No. 5, 2018, pp. 185-193. doi: 10.11648/j.ajmp.20180705.13

Received: November 10, 2016; Accepted: November 2, 2018; Published: December 5, 2018

\begin{abstract}
This model describes the production and destruction mechanism of nitrogen plasma at atmospheric pressure. We have studied the mechanisms of chemical dissociation, ionization, ion conversion and recombination in nitrogen plasmas, with kinetic temperature $\left(T_{e}\right)$ of the free electrons being higher than the kinetic temperature $\left(T_{g}\right)$ of heavy species. Therefore, the investigation of nitrogen plasma species in a wide range of pressure from 1 Torr to 760 Torr is interesting phenomena for obtaining the equilibrium state when the nitrogen species breakdown. In order to calculate the species densities to reach thermodynamic equilibrium under various conditions, a set of chemical kinetic reactions of nitrogen under consideration have been simulated. It solves the particle balance equations for a set of interacting species. In this study 16 reactions and 4 species of Nitrogen $\mathrm{N}, \mathrm{N}_{2}, \mathrm{~N}^{+}, \mathrm{N}_{2}^{+}$and electron have been considered. The densities of the charged and neutral species are modeled by continuity equations which includes the relevant plasma-chemical kinetics. Nitrogen species density is guided by continuity equation where chemical processes and Arrhenius form are used to follow the change of species density over the time. To calculate the species densities over pressure, temperature and time the continuity equations of the 16 reactions for the 5 species under consideration giving their initial pressure, densities and temperatures, with the latter held constant have been solved. The variations of species densities have been investigated as a function of pressure ranging from 1 to 760 Torr. This model shows that as the pressure is increased the species densities of nitrogen plasma also increase from pressure 1 to 200 Torr and after pressure above 200 Torr the species densities become almost saturated. The change of species densities at various temperatures ranging from 2000 Kelvin to 25000 Kelvin is successfully investigated. The destruction and production rates of the nitrogen species also have been calculated within the time ranging from 0 to $19 \mathrm{nS}$ and it shows that the density of nitrogen plasma increases with time. In our study we have considered the gas and electron temperature as $10 \mathrm{k}$ Kelvin and $4 \mathrm{eV}$ respectively.
\end{abstract}

Keywords: Classification of Plasma, Simulation of Nitrogen Plasma, Reaction Rate and Rate Coefficient, Ionization Process, Plasma Modeling, Fluid Modeling Approach

\section{Introduction}

When two particles collide, various phenomena may occur [1]. As examples, one or both particles may change their momentum or their energy, neutral particles can become ionized, and ionized particles can become neutral. We introduce the fundamentals of collisions between electrons, positive ions, and gas atoms in this chapter. For electrons colliding with atoms, the main processes are elastic scattering in which primarily the electron momentum is changed, and inelastic processes such as excitation and ionization. For ions colliding with atoms, the main processes are elastic scattering in which momentum and energy are exchanged, and resonant charge transfer. Other important processes occur in molecular gases. These include dissociation, dissociative recombination, processes involving negative ions, such as attachment, detachment, and positive-negative ion charge transfer, and processes involving excitation of molecular vibrations and rotations.

\subsection{Plasma Temperature}

Plasma contains a mixture of particles with different electric charges and masses [2]. At a first approximation, the plasma may be considered, thermally, as consisting of two 
systems: the first containing only electrons and the second containing the heavy species, that is, neutral atoms or molecules, ions, and neutral molecular fragments. The electrons gain energy from the electric field, which energizes the plasma, and lose part of it by transfer to the second system through elastic or inelastic collisions. The system of heavy particles loses energy to the surroundings, either by radiation or by heat transfer to the walls of the vessel containing the plasma. The electrons and the heavy species in the plasma can be considered approximately as two subsystems, each in its own thermal quasi-equilibrium. The ions and electrons in the plasma can therefore be characterized by their specific different average temperatures: the ion temperature ion, $T_{i}$ and the electron temperature $T_{e}$ [3]. Actually in some cases additional temperatures may characterize the particles in the plasma. $T_{e x}$ which characterizes the energy of the excited particles in the plasma; the ionization temperature $T_{i o n}$; the dissociation temperature $T_{d i s}$, which characterize the energy of ionization and dissociation; and the radiation temperature $T_{\text {rad }}$, which characterizes the radiation energy [4]. Thermodynamic equilibrium will exist in the plasma only if the following equation is satisfied: $T_{g}=T_{e x}=T_{i o n}=T_{\text {dis }}=T_{\text {rad }}=T_{e}$

\subsection{Plasma Parameters}

The plasma, especially one sustained in a mixture of molecular gases, contains a multitude of different neutral and charged particles. A group of identical particles in the plasma is commonly referred to as a species. The plasma is broadly characterized by the following basic parameters [5]: The density of the neutral particles $n_{g}$, the densities of electrons and ions are $n_{e}$ and $n_{i}$. In the quasi-neutral state of plasma the densities of the electrons and of the ions are usually equal $n_{i}=n_{e}=n$ and $n$ is called the plasma density.

\subsection{Electron Temperature}

The average energy of the electrons is related to their temperature by [6]: $w_{a v}=\frac{3}{2} \kappa T_{e}$. Where $w_{a v}$ is the average energy of the electron. At low pressures, the electron temperature is much greater than the temperature of the gas, $T_{e} \gg T_{g}$. When the pressure in the plasma increases, the energy transfer from electron to neutrals increases, causing an increase in the temperature of the gas and decrease of the electron temperature

\subsection{Plasma Classification}

Plasmas are described by many characteristics, such as temperature, pressure, degree of ionization, and density, the magnitude of which, and approximations of the model describing them, gives rise to plasmas that may be classified in different ways.: Depending on pressure: Plasmas can be classified depending on pressure are: i) Low pressure plasma: When the pressure of the plasma is less than one Torr then the plasma is called the low pressure plasma [7]. ii) Intermediate pressure or medium pressure plasma: Intermediate pressure or medium pressure plasma is one in which the pressure of the plasma lies in the range 1 Torr to 40
Torr [8]. iii) High pressure plasma: The pressure of the plasma is in the range between pressures 40 Torr to 700 Torr then this plasma is called the high pressure plasma [9]. iv) Atmospheric plasma: When the pressure range of the plasma is above 700 Torr then it is called the Atmospheric plasma [10].

\subsection{Simulation of Nitrogen Plasma}

The equilibrium process of nitrogen plasma species by chemical kinetic reactions along various Pressure and temperature is successfully investigated. The equilibrium process is required in industrial Application to obtain the stable condition when heating up the material for having homogenous Reaction. Nitrogen species densities is obtained by solving the continuity equations [11] or rate balance equations and extended Arrhenius forms [12] are used to obtain the rate coefficient of the species for the chemical kinetic reactions of consideration. These equations are used to integrate the change of species density over the time, pressure and temperature. The integration is to acquire species density and the reaction rate of each reaction where pressure, temperature and time dependence are imposed. It is important to understand the chemical mechanism of dissociation, ionization, and recombination in nitrogen plasmas, with kinetic temperature of the free electrons (Te) being higher than the kinetic temperature of heavy species $\left(T_{g}\right)$. The most distinct feature that distinguishes nonequilibrium discharge plasmas from other discharge plasmas is the electron temperature $\left(T_{e}\right)$, i.e. average electron energy in the plasmas is about two orders of magnitude higher than ion temperature $\left(T_{i}\right)$ and neutral or gas temperature $\left(T_{g}\right)$. Therefore, the investigation of nitrogen plasma species in a wide range of pressure from low to higher pressure is interesting phenomena for obtaining the equilibrium state when the nitrogen species breakdown. In order to calculate the species densities to reach thermodynamic equilibrium under various conditions, a simple chemical kinetic reaction has been simulated. It solves the rate balance equations for a set of interacting species. In our work, we have investigated the behavior of nitrogen species densities at a given initial pressure, density and temperature. In order to investigate the equilibrium state a set of reaction set of nitrogen plasma is introduced. The rate of reaction is calculated to investigate how fast a reaction process occurs. The concept of rate coefficient is used in a similar fashion to describe reactive collisions between the randomly moving heavy particles, where the reaction probability is determined by the relative velocity between the colliding heavy particles. At equilibrium conditions, the temperature dependence of the rate coefficient can be described by an Arrhenius equation. The most dominant reaction rates are not only required to demonstrate the thermodynamic equilibrium process but also to determine significant species densities in contributing the combustion process of the material. In this purpose, four nitrogen species; $N, N^{+}, N_{2}, N_{2}^{+}$and electron are chosen. The integration of chemical kinetic model and the reaction rates for atmospheric pressure discharge is investigated. 


\section{Reaction Rate and Reaction Rate Coefficients}

The number of elementary processes $\omega$ that take place in unit volume per unit time is called the elementary reaction rate [13]. In principal this concept can be used for any kind of reaction: monomolecular, bimolecular, and three-body. For bimolecular processes $A+B$, the reaction rate can be calculated by multiplying the interaction frequency of partner $\mathrm{A}$ with partner $\mathrm{B} \mathrm{v}_{\mathrm{A}}$, and by the number of particles $\mathrm{A}$ in the unit volume (which is their number density $n_{A}$ ) [14]: $\omega_{\mathrm{A}+\mathrm{B}}=v_{\mathrm{A}} \mathrm{n}_{\mathrm{A}}=\langle\sigma v\rangle \mathrm{n}_{\mathrm{A}} \mathrm{n}_{\mathrm{B}}$.

\subsection{Ionization Process}

The key process in plasma is ionization because it is responsible for plasma generation: birth of new electrons and positive ions [15]. The simplest ionization process-the ionization by electron-impact. In general, all ionization processes can be subdivided into the following five groups: i) Direct ionization by electron impact: It includes the ionization of neutrals: preliminary not-excited atoms, radicals, or molecules by an electron whose energy is sufficiently large to provide the ionization act in one collision [16]. The collision between atomic nitrogen and electron is an example of direct ionization by electron impact. $N+e \rightarrow$ $N^{+}+2 e$. Stepwise ionization by electron impact: It includes the ionization of preliminary excited neutral species. These processes are important mainly in thermal or energy-intense discharges, when the degree of ionization (ratio of number densities of electrons and neutrals) and the concentration of highly excited neutral species are quite large. The first electron-neutral collisions result in forming highly excited species, and then a final collision with a relatively low energy electron provides the actual ionization act [14]. $N^{+}+e+$ $e \rightarrow N^{*}+e \rightarrow N+e$

ii) Electron impact ionization: In electron impact ionization the primary incident electron removes an electron from the atom or molecule, producing a positive atomic or molecular ion and two electrons, e.g.

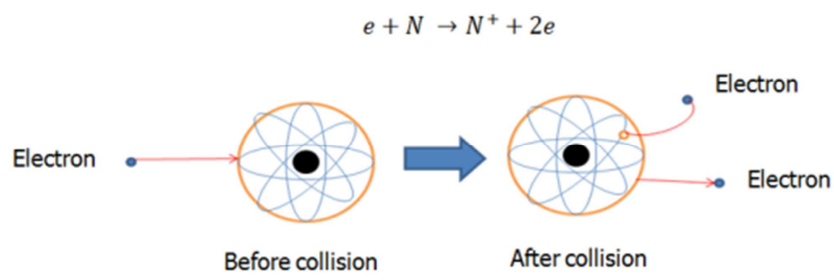

Figure 1. Electron impact ionization

iii) Associative ionization: This process is discovered by Hornbeck and Molnar in 1951 [17]. It is important in the inert gases and for nitrogen the it can be represented as: $N+N^{*} \rightarrow N_{2}^{+}+e$. The electrons are free to move with the release of small binding energy of $\sim 1 \mathrm{eV}$ in association with an ion and an atom into a molecular ion.

iv) Dissociation: The process of dissociation is the breaking apart of a molecule. An nitrogen molecule can be dissociated into two nitrogen atoms, but an atomic gas such as argon cannot be dissociated at all. $e+N_{2} \rightarrow e+N+N$ v) Ion conversion: When the positive ions collide with the neutral gas atoms, the gas atoms are converted into molecular gas ions as well as neutral gas atoms [18], which can be represented by the following collision process: $N^{+}+N_{2} \rightarrow$ $N_{2}^{+}+N$.

\subsection{Recombination}

Recombination is to ionization electron coalesces with a positive ion to form a neutral atom [19]. Assume that the electron has a mass $\mathrm{m}$ and has a velocity, relative to the ion mass $\mathrm{M}$, of $\mathrm{v}$ before recombination. Let their joint velocity after coalescence be $u$. The potential energy of the atom has decreased by $U_{i}$, the relevant ionization energy. Then, to continue our earlier applied mathematical exercises: Conservation of momentum: $m v=(m+M) u$.Conservation of energy: $\frac{1}{2} m v^{2}=\frac{1}{2}(m+M) u^{2}-U_{i}$.This process is represented as $N^{+}+e \rightarrow N+2 \mathrm{e}$

\subsubsection{Dissociative Recombination}

The dissociative recombination occurs as a very fast process in the weakly ionized plasma and can be written as $N^{+}+e+e \rightarrow N+e$. The electron-ion recombination is the reverse process of ionization, i.e., an electron combines with appositive ion to form a neutral atom. There are different types of channels in the atmospheric pressure discharges for the dissociative recombination. $N_{2}^{+}+e \rightarrow$ $N^{*}+N$. When the nitrogen ion is molecular, a two-body recombination process is possible because the collision product can dissociate and the recombination energy is converted into kinetic and potential energy of the dissociation products [20].

\subsubsection{Three-Body Ion-Ion Recombination}

The neutralization process at moderate and high pressures ( $p>10-30$ torr) effectively proceeds via a triple collision of a heavy neutral with a negative and a positive ion [21]: $A^{-}+B^{+}+M \rightarrow A+B$. This process dominates the ion-ion recombination at moderate and high pressures $(\mathrm{p}>10$ to 30 torr). The three-body reaction has the third kinetic order only in the moderate pressure range, usually less than 1 atm (for high pressures, the process is limited by ion mobility). This means that only at moderate pressures is the reaction rate proportional to the product of concentrations of all three collision partners, positive and negative ions, and neutrals.

\subsubsection{Electron-Ion Recombination Process}

The ionization processes are as a source of electrons and positive ions, e.g., as a source of plasma generation [22]. Conversely, the principal loss mechanisms of charged particles, the elementary processes of plasma degradation. Obviously, the losses together with the ionization processes determine a balance of charge particles and plasma density. The variety of channels of charged particle losses can be subdivided into three qualitatively different groups. The first group includes different types of electron-ion recombination processes, in which collisions of the charged particles in a discharge volume lead to their mutual neutralization. Recombination energy in these processes goes into dissociation of the intermediately formed molecule ion and to 
excitation of the dissociation products. These processes are common for molecular gases, but they can also be important in atomic gases because of the formation of molecular ions in the ion conversion processes: $N^{+}+N+N \rightarrow N^{2+}+$ $N$.Two-body recombination for atomic ions or for molecular ions that do not subsequently dissociate can only occur with emission of a photon: $e+N^{+} \rightarrow N+h v$. The rate constants are typically three to five orders of magnitude lower than for dissociative recombination. Electron-ion neutralization is due to a three-body electron-ion recombination in atomic gases in the absence of molecular ions: $e+e+N^{+} \rightarrow$ $N^{*}+e$. Energy excess in this case is going into the kinetic energy of a free electron, which participates in the recombination act as "a third-body partner. Finally, the recombination energy can be converted into radiation in the process of radiative electron-ion recombination [23]: $e+$ $N^{+} \rightarrow N^{*} \rightarrow N+\hbar \omega$.

\subsection{Collision}

A collision is an isolated event in which two or more moving bodies (colliding bodies) exert forces on each other for a relatively short time. Although the most common colloquial use of the word "collision" refers to accidents in which two or more objects collide, the scientific use of the word "collision" implies nothing about the magnitude of the forces.

\subsubsection{Three Body Collision}

A third body takes part in the collision process, and the third body allows the recombination process to simultaneously satisfy the conservation requirements of energy and momentum. The third body often a wall, ubiquitous in our plasma processes, or it may be another gas atom. The probability of the gas atom taking part in the process will increase with increasing pressure [24]. $\mathrm{N}^{+}+$ $N_{2}^{+}+N_{2} \rightarrow N_{3}^{+}+N_{2}$.

\subsubsection{Ion-Neutral Collisions}

Ions and neutrals can collide with each other elastically or inelastically to either exchange charges or cause further ionization. $\mathrm{N}_{2}^{+}+N \rightarrow N^{+}+N_{2}$.

\subsubsection{Charge Transfer Collisions}

The probability of a collision leading to the exchange of charge, generically known as charge transfer, is usually greater for atomic ions moving in parent atoms (symmetrical resonant charge transfer), e.g. $N+N^{+} \rightarrow N+N^{+}$.

\subsection{Electron Attachment}

There is a possibility that an electron colliding with an atom may join on to the atom to form a negative ion. This process is known as electron attachment.

Three-Body Electron Attachment which attachment processes of formation of negative ions in the collision of an electron with two heavy particles (at least one of which is supposed to have positive electron affinity) can be shown as [25]: $N_{2}^{+}+e+N_{2} \rightarrow N_{2}+N_{2}$.

\section{Plasma Modeling}

A most general classification of plasma models is: i) Models relying on experimental measurements ii) Selfconsistent models.

\subsection{Models Based on Experimental Measurements}

These models (i.e. voltage, current (total ion, electron), impedance, electron density, electron temperature, ion flux, excited or ground-state species density, consumption of gas precursors, deposition rate etc) are used as model inputs.

\subsection{Self-Consistent Models}

It require as input, only the applied voltage. They have the advantages that can be applied to any system if a specific geometry is given and that they handle all sub-processes. "Experimental" or fully self-consistent glow discharge models can be further classified, according to the mathematical approach for the solution of the problem, to the following types: i) Analytical models [26-28] ii) Numerical models.

\subsection{Numerical Models}

These models are generally more popular in self consistent plasma modeling. They can be further distinguished, according to the methodology used for the management of electron and ion transport in RF discharges, in the following categories: i) kinetic model ii) Fluid Model iii) Hybrid Model

\subsubsection{Kinetic Modeling Approach}

Kinetic models describe the distribution function of particle velocity at each point in the discharge plasma. It Particle modeling is useful method to extract kinetic information, such as distribution function, reaction-rates, etc., self-consistently and to study the transport of particles across the discharge volume simultaneously. Kinetic models typically involve the solution of the Boltzmann equation for the species velocity or energy distribution function in both space and time or the particle simulations [29-31] using techniques such as Monte Carlo methods. Although the Boltzmann equation can provide much more information than the continuum fluid model, its solution is computationally intensive requiring an order of magnitude more computer time than the fluid calculation as kinetic model are inherently higher dimensional and the solutions are functions of the velocity, space and time, the additional degrees of freedom being described in the full phase space of the problem. The coupling between the Boltzmann equation and the Maxwell's equations for electrostatics is a difficult numerical problem as the particle dynamics should include the strong body force effect of electrostatics. The solution variable in the kinetic models is the species velocity distribution function. Kinetic model involves solving the continuity equation.

Continuity Equation: Neutral: $\frac{\partial \mathrm{n}}{\partial \mathrm{t}}=\mathrm{r}_{\mathrm{i}} \mathrm{n}_{\mathrm{c}_{\mathrm{i}}}-\mathrm{r}_{\mathrm{j}} \mathrm{n}_{\mathrm{d}_{\mathrm{j}}}$, Ions: $\frac{\partial n_{i}}{\partial t}=r_{i} n_{c_{i}}-r_{j} n_{d_{j}}$ 


\subsubsection{Fluid Modeling Approach}

Fluid models generally consist of a few moments of the Boltzmann equation for the various species with an assumed distribution function to describe the macroscopic quantities, such as species density, momentum and electron mean energy [29-31]. The space and time variations of these quantities provide the structure of the macroscopic quantities. The implementation of secondary electron emission coefficient is considered as the boundary condition in the fluid model.

\subsubsection{Hybrid Modeling Approach}

Hybrid models [31-32] use the kinetic approach in order to handle the non-local transport of electrons and ions in the discharges and to derive transport coefficients of charged species.

\section{Chemistry}

We have consider particle and energy balance for two basic types of discharges: electropositive discharge and electronegative discharge. In electropositive discharges, there are only two species that are normally considered, electrons and positive ion. But in electronegative discharges, there are three species that are normally considered, electrons, one positive ion and one negative ion species. The simulated discharge gas is nitrogen and it is described using 5 chemical species and 16 chemical reactions. The species included in this model are electrons, $\mathrm{N}, \mathrm{N}^{+}, \mathrm{N}_{2}$ and $\mathrm{N}_{2}^{+}$. The reactions that are described in this model define the production and loss processes of the different species in the discharge. In our study the following 16 reactions of nitrogen are taken into account.

i) $N_{2}+N_{2} \stackrel{k_{1}}{\rightarrow} \mathrm{N}+\mathrm{N}+\mathrm{N}_{2}$

ii) $N_{2}+N \stackrel{k_{2}}{\rightarrow} N+N+N$

iii) $N+N \stackrel{k_{3}}{\rightarrow} N_{2}^{+}+e$

iv) $N^{+}+N_{2} \stackrel{k_{4}}{\rightarrow} N_{2}^{+}+N$

v) $N_{2}+N_{2} \stackrel{k_{5}}{\rightarrow} N_{2}^{+}+N_{2}+e$

vi) $N+e \stackrel{k_{6}}{\rightarrow} N^{+}+2 e$

vii) $N_{2}+e \stackrel{k_{7}}{\rightarrow} N+N+e$

viii) $N_{2}+e \stackrel{k_{8}}{\rightarrow} N_{2}^{+}+2 e$

ix) $N_{2}^{+}+e \stackrel{k_{9}}{\rightarrow} N^{+}+N+e$

x) $\mathrm{N}_{2}^{+}+N \stackrel{k_{10}}{\longrightarrow} N_{2}+N^{+}$

xi) $N_{2}^{+}+e \stackrel{k_{11}}{\longrightarrow} N+N$

xii) $N^{+}+N+N_{2} \stackrel{k_{12}}{\rightarrow} N+N+N_{2}$

xiii) $N_{2}^{+}+N_{2}+e \stackrel{k_{13}}{\longrightarrow} N_{2}+N_{2}$

xiv) $N_{2}^{+}+N+e \stackrel{k_{14}}{\longrightarrow} N+N_{2}$

xv) $N^{+}+N_{2}+e \stackrel{k_{15}}{\longrightarrow} N+N_{2}$

xvi) $N^{+}+N+e \stackrel{k_{16}}{\longrightarrow} N+N$

Calculation of the Rate Coefficients of the Reactions.

\subsection{Dissociation: Dissociation of Molecular Nitrogen}

Dissociation $\mathrm{N}_{2}$ of by heavy particle impact: The collisions between two molecular nitrogen dissociate one of the nitrogen molecules as seen by the following reaction: $\mathrm{N}_{2}+\mathrm{N}_{2} \stackrel{\mathrm{k} 1}{\rightarrow} \mathrm{N}+\mathrm{N}+\mathrm{N}_{2}$. The rate coefficient $\mathrm{k}_{1}$ is calculated [33] by the following expression: $5.0 \times 10^{-8} \times \mathrm{T}^{-1.6} \exp \left(-\frac{113260}{\mathrm{~T}}\right)$, where the $\mathrm{T}$ is the gas temperature in Kelvin.

i) Dissociation of molecular nitrogen by atomic nitrogen impact: The collision between atomic and molecular nitrogen dissociates the molecular nitrogen as: $\mathrm{N}_{2}+\mathrm{N} \stackrel{\mathrm{k} 2}{\rightarrow} \mathrm{N}+\mathrm{N}+$ N.The rate coefficient $\mathrm{k}_{1}$ of the above process is calculated by the following expression: $1.17 \times 10^{-8} \times \mathrm{T}^{-1.6} \exp \left(-\frac{113260}{\mathrm{~T}}\right)$, where the $\mathrm{T}$ is the gas temperature in Kelvin.

ii) Dissociation of $\mathrm{N}_{2}$ by electron impact: $\mathrm{N}_{2}+\mathrm{e} \stackrel{\mathrm{k} 7}{\rightarrow} \mathrm{N}+$ $\mathrm{N}+\mathrm{e}$. The rate coefficient $\mathrm{k}_{7}$ of the above process is calculated by the following expression: $4.11 \times 10^{-33} \mathrm{~T}^{6.16} \exp \left(\frac{-113263.0}{\mathrm{~T}}\right)$,where $\mathrm{T}$ is the electron temperature in eV.Dissociation of molecular nitrogen ion by electron impact: $\mathrm{N}_{2}^{+}+\mathrm{e} \stackrel{\mathrm{k} 9}{\rightarrow} \mathrm{N}^{+}+\mathrm{N}+\mathrm{e}$. The rate coefficient $\mathrm{k}_{9}$ of the above process is calculated [34] by the following expression:

$8.02 \times$ $10^{-31}(\mathrm{~T})^{5.54} \exp \left(-\frac{101117.1}{\mathrm{~T}}\right)$ where $\mathrm{T}$ is the electron temperature in $\mathrm{eV}$.

iii) Ion conversion by heavy particle: $\mathrm{N}^{+}+\mathrm{N}_{2} \stackrel{k 4}{\rightarrow} \mathrm{N}_{2}^{+}+$ $N, N_{2}^{+}+N \stackrel{k 10}{\rightarrow} N^{+}+N_{2}$.

The rate coefficient $k_{4}$ and $k_{10}$ of the above two processes were calculated by the following expression: $2.024 \times$ $10^{-19} T^{2.06} \exp \left(-\frac{30057.0}{T}\right)$, where $\mathrm{T}$ is the gas temperature in Kelvin.

\subsection{Ionization}

Ionization of $N_{2}$ by electron impact: $N_{2}+e \stackrel{k 8}{\rightarrow} N_{2}^{+}+2 e$.

The rate coefficient $k_{8}$ of the above two processes were calculated by the following expression:

$8.58 \times 10^{-12} \times T^{0.72} \exp \left(-\frac{184300.2}{T}\right)$ where $\mathrm{T}$ is the electron temperature in eV. i) Ionization of $N$ by direct electron impact: $N+e \stackrel{k_{6}}{\rightarrow} N^{+}+e+e$. The rate coefficient $k_{6}$ of the above two processes were calculated by the following expression: $4.17 \times 10^{3} \times T^{0} \exp \left(-\frac{168200}{T}\right)$, where $\mathrm{T}$ is the electron temperature in $\mathrm{eV}$. Ionization of $N_{2}$ by heavy particle impact: $N_{2}+N_{2} \stackrel{k 5}{\rightarrow} N_{2}^{+}+N_{2}+e$. The rate coefficient $k_{6}$ of the above two processes were calculated [34] by the expression: $4.74 \times 10^{-22} \times T^{2.00} \exp \left(-\frac{180640}{T}\right)$, where $\mathrm{T}$ is the gas temperature in Kelvin.

\subsection{Three Body Electron-Ion Recombination}

Three body Recombination of molecular nitrogen ion $\left(\mathrm{N}_{2}^{+}\right)$ and electron by heavy particle $\left(N_{2}\right)$ impact: $N_{2}^{+}+N_{2}+e$ $\stackrel{k 13}{\longrightarrow} N_{2}+N_{2}$. The rate coefficient $k_{13}$ is calculated by the following [33] expression:6.07 $\times 10^{-34} \times T^{-2.5}$, where $\mathrm{T}$ is 
the electron temperature in eV.Three body Recombination of molecular nitrogen ion $\left(N_{2}^{+}\right)$and electron by heavy particle $(N)$ impact: $N_{2}^{+}+N+e \stackrel{k 14}{\longrightarrow} N+N_{2}$. The rate coefficient $k_{14}$ is calculated by the following expression: $6.07 \times 10^{-34} \times T^{-2.5}$. Recombination of nitrogen ion $\left(N^{+}\right)$ and electron by heavy particle $\left(N_{2}\right)$ impact: $N^{+}+N_{2}+e$ $\stackrel{k 15}{\longrightarrow} N+N_{2}$. The rate coefficient $k_{15}$ is calculated by the following expression: $6.07 \times 10^{-34} \times T^{-2.5}$. Recombination of $N^{+}$and electron by heavy particle $(N)$ impact: $N^{+}+N+$ $e \stackrel{k 16}{\longrightarrow} N+N$. The rate coefficient $k_{16}$ is calculated by the following expression: $1.66 \times 10^{-35} \times T^{-2.5}$.

\section{Kinetics Model Equations}

The reactions are elementary, and can be assembled into a model for intrinsic chemical kinetics for the concentration of these nine species. If the reactor is assumed kinetics dominated (well mixed) — when there action time is much shorter than the ambipolar diffusion time, then each of the elementary reactions contributes mass action law terms to the kinetics model with rate constants, with number densities defined as: $N_{N}$ is the density of $N ; N_{N_{2}}^{+}$is the density of $N_{2}^{+} ; \mathrm{N}_{\mathrm{N}^{+}}$is the density of $N^{+} ; N_{e}$ is the density of $e$ and $N_{N_{2}}$ is the density of $N_{2}$.

The Rate Balance Equation for the Reactions

i) $\frac{d N_{2}}{d t}=k_{10} N_{N_{2}^{+}} N_{N}+k_{13} N_{N_{2}^{+}} N_{N_{2}} N_{e}+k_{14} N_{N_{2}^{+}} N_{N} N_{e}-$ $2 k_{1} N_{N_{2}} N_{N_{2}}-2 k_{2} N_{N_{2}} N_{N}-k_{4} N_{N^{+}}-k_{5} N_{N_{2}^{+}} N_{N_{2}}-$ $k_{7} N_{N_{2}} N_{e}-k_{8} N_{N_{2}} N_{e}$

ii) $\frac{d N}{d t}=2 k_{1} N_{N_{2}} N_{N_{2}}+2 k_{2} N_{N_{2}} N_{N}+k_{4} N_{N^{+}} N_{N_{2}}+$ $2 k_{7} N_{N_{2}} N_{e}+k_{9} N_{N_{2}^{+}} N_{e}+2 k_{11} N_{N_{2}^{+}} N_{e}+k_{12} N_{N_{2}} N_{N} N_{N^{+}}+$ $k_{15} N_{N_{2}} N_{N}+N_{e}+k_{16} N_{N}+N_{e} N_{N}-k_{3} N_{N} N_{N}-k_{6} N_{N} N_{e}-$ $k_{10} N_{N_{2}}+N_{N}$

iii) $\frac{d N_{2}^{+}}{d t}=k_{3} N_{N} N_{N}+k_{4} N_{N^{+}} N_{N_{2}}+k_{5} N_{N_{2}} N_{N_{2}}+$ $k_{8} N_{N_{2}} N_{e}-k_{9} N_{N_{2}^{+}} N_{e}-k_{10} N_{N_{2}^{+}} N_{N}-k_{11} N_{N_{2}^{+}} N_{e}-$ $k_{13} N_{N_{2}^{+}} N_{N} N_{e}-k_{14} N_{N_{2}^{+}} N_{N} N_{e}$

iv) $\frac{d N^{+}}{d t}=k_{6} N_{N} N_{e}+k_{9} N_{N_{2}^{+}} N_{e}+k_{10} N_{N_{2}^{+}} N_{N}-$ $k_{4} N_{N^{+}} N_{N_{2}}-k_{12} N_{N_{2}} N_{N} N_{N^{+}}-k_{15} N_{N_{2}} N_{N^{+}} N_{e}$ $k_{16} N_{N^{+}} N_{e} N_{N}$

v) $\frac{d e}{d t}=k_{3} N_{N} N_{N}+k_{5} N_{N_{2}} N_{N_{2}}+k_{6} N_{N} N_{e}+k_{8} \mathrm{~N}_{\mathrm{N}_{2}} \mathrm{~N}_{\mathrm{e}}-$ $\mathrm{k}_{11} \mathrm{~N}_{\mathrm{N}_{2}^{+}} \mathrm{N}_{\mathrm{e}}-\mathrm{k}_{13} \mathrm{~N}_{\mathrm{N}_{2}^{+}} \mathrm{N}_{\mathrm{N}} \mathrm{N}_{\mathrm{e}}-\mathrm{k}_{14} \mathrm{~N}_{\mathrm{N}_{2}^{+}} \mathrm{N}_{\mathrm{N}} \mathrm{N}_{\mathrm{e}}-$ $\mathrm{k}_{15} \mathrm{~N}_{\mathrm{N}_{2}} \mathrm{~N}_{\mathrm{N}}+\mathrm{N}_{\mathrm{e}}-\mathrm{k}_{16} \mathrm{~N}_{\mathrm{N}}+\mathrm{N}_{\mathrm{e}} \mathrm{N}_{\mathrm{N}}$

\section{Results and Discussions}

\subsection{Simulation Techniques: Steady State Solution Technique}

There are various techniques for solving the steady state system of non-linear equations [36]. These techniques are: i) Gauss-Newton Method ii) Basics of the Finite Element Method iii) Adaptmesh (Adaptive mesh generation and PDE solution) The functions that can be used to obtain a steady state solution are: i) fminunc (Find minimum of unconstrained multivariable function) ii). fsolve (Solve system of nonlinear equations) iii). lsqlin (Solve constrained linear least-squares problems) iv). quadprog (Solve quadratic programming problems)

\subsection{Steady State Equations}

The species density is a function of pressure and temperature. The steady state rate balance is needed to solve to simulate the behavior the species densities. Continuity Equation: Neutral: $\frac{\partial \mathrm{n}}{\partial \mathrm{t}}=\mathrm{r}_{\mathrm{i}} \mathrm{n}_{\mathrm{c}_{\mathrm{i}}}-\mathrm{r}_{\mathrm{j}} \mathrm{n}_{\mathrm{d}_{\mathrm{j}}}$, Ions: $\frac{\partial \mathrm{n}_{\mathrm{i}}}{\partial \mathrm{t}}=\mathrm{r}_{\mathrm{i}} \mathrm{n}_{\mathrm{c}_{\mathrm{i}}}-\mathrm{r}_{\mathrm{j}} \mathrm{n}_{\mathrm{d}_{\mathrm{j}}} \quad$. These continuity equations are called steady state equations when the time dependent terms of it is zero. Now we can write the steady state rate balance equations from the reactions and rate coefficients:

i) $k_{10} N_{N_{2}^{+}} N_{N}+k_{13} N_{N_{2}^{+}} N_{N_{2}} N_{e}+k_{14} N_{N_{2}^{+}} N_{N} N_{e}-$ $2 k_{1} N_{N_{2}} N_{N_{2}}-2 k_{2} N_{N_{2}} N_{N}-k_{4} N_{N^{+}}-k_{5} N_{N_{2}^{+}} N_{N_{2}}-$ $k_{7} N_{N_{2}} N_{e}-k_{8} N_{N_{2}} N_{e}=0$

ii) $k_{1} N_{N_{2}} N_{N_{2}}+2 k_{2} N_{N_{2}} N_{N}+k_{4} N_{N}+N_{N_{2}}+2 k_{7} N_{N_{2}} N_{e}+$ $k_{9} N_{N_{2}^{+}} N_{e}+2 k_{11} N_{N_{2}^{+}} N_{e}+k_{12} N_{N_{2}} N_{N} N_{N^{+}}+$ $k_{15} N_{N_{2}} N_{N^{+}} N_{e}+k_{16} N_{N}+N_{e} N_{N}-k_{3} N_{N} N_{N}-k_{6} N_{N} N_{e}-$ $k_{10} N_{N_{2}^{+}} N_{N}=0$

iii) $k_{3} N_{N} N_{N}+k_{4} N_{N^{+}} N_{N_{2}}+k_{5} N_{N_{2}} N_{N_{2}}+k_{8} N_{N_{2}} N_{e}-$ $k_{9} N_{N_{2}^{+}} N_{e}-k_{10} N_{N_{2}^{+}} N_{N}-k_{11} N_{N_{2}^{+}} N_{e}-k_{13} N_{N_{2}^{+}} N_{N} N_{e}-$ $k_{14} N_{N_{2}^{+}} N_{N} N_{e}=0$

iv) $k_{6} N_{N} N_{e}+k_{9} N_{N_{2}^{+}} N_{e}+k_{10} N_{N_{2}^{+}} N_{N}-k_{4} N_{N^{+}} N_{N_{2}}-$ $k_{12} N_{N_{2}} N_{N} N_{N^{+}}-k_{15} N_{N_{2}} N_{N^{+}} N_{e}-k_{16} N_{N^{+}} N_{e} N_{N}=0$

v) $k_{3} N_{N} N_{N}+k_{5} N_{N_{2}} N_{N_{2}}+k_{6} N_{N} N_{e}+k_{8} N_{N_{2}} N_{e}-$ $k_{11} N_{N_{2}^{+}} N_{e}-k_{13} N_{N_{2}^{+}} N_{N} N_{e}-k_{14} N_{N_{2}^{+}} N_{N} N_{e}-$ $k_{15} N_{N_{2}} N_{N}+N_{e}-k_{16} N_{N}+N_{e} N_{N}=0$

We used fsolve and lsqlin functions to solve the above system of non-linear equations. Here we used the MATLAB version mathlab 7.6.0.324(R2008a). fsolve function is part of MATLAB's optimization toolbox and, according to the documentation. The MATLAB routine fsolve is used to solve sets of nonlinear algebraic equations. Either fsolve or lsqlin MATLAB (version 7.6.0.324(R2008a)) functions can be used to solve the above system of non-linear equations. The values of all the rate coefficients were entered into the following function in order to use MATLAB $f$ solve function. The species density is normalized by the gas density $N_{N_{2}}$. The gas density $N_{N_{2}}$ is calculated as [1]; $N_{N_{2}}=\frac{P}{\kappa_{B} * T_{g}}$ Where $P$ is the pressure in Torr and $\kappa_{B}$ is the Boltzmann constant in $J k^{-1}$. All the rate coefficients are normalized as: $k_{i}^{*}=\frac{k_{i}}{r_{m}}$ Where $i=1-16$. And $r_{m}$ is the maximum value of the rate coefficient.As a first step, we have performed simulations of a nitrogen discharge. The chemical kinetics is clearly much simplified there and nitrogen plasma is the main product of the discharge. By solving the steady state rate balance equations we have calculated the species density to investigate the effect of pressure and temperature on the plasma composition.

\subsection{Result}

In our present kinetic model, some of the chemical species 
of nitrogen are taken into account. The nitrogen plasma employed in our study includes 5 species ( electron, $\mathrm{N}_{2}, \mathrm{~N}_{2}^{+}, \mathrm{N}, \mathrm{N}^{+}$) and 16 reactions. This set of nitrogen plasma includes (1) direct ionization (i) by electron impact and (ii) heavy particle impact $\left(\mathrm{N}_{2}, \mathrm{~N}\right)$ impact, (2) dissociative ionization, (3) dissociation (4) ion conversion (5) three body electron ion recombination. To show the various species densities under pressure, temperature and time all of the rate balance equations of nitrogen species under consideration are solved.

\subsubsection{Pressure Dependent}

Figure-2 shows the Pressure dependent species densities in nitrogen plasma at gas temperature and electron temperature $\mathrm{T}_{\mathrm{g}}=10 \mathrm{k}$ kelvin and $\mathrm{T}_{\mathrm{e}}=4.0 \mathrm{eV}$ respectively.

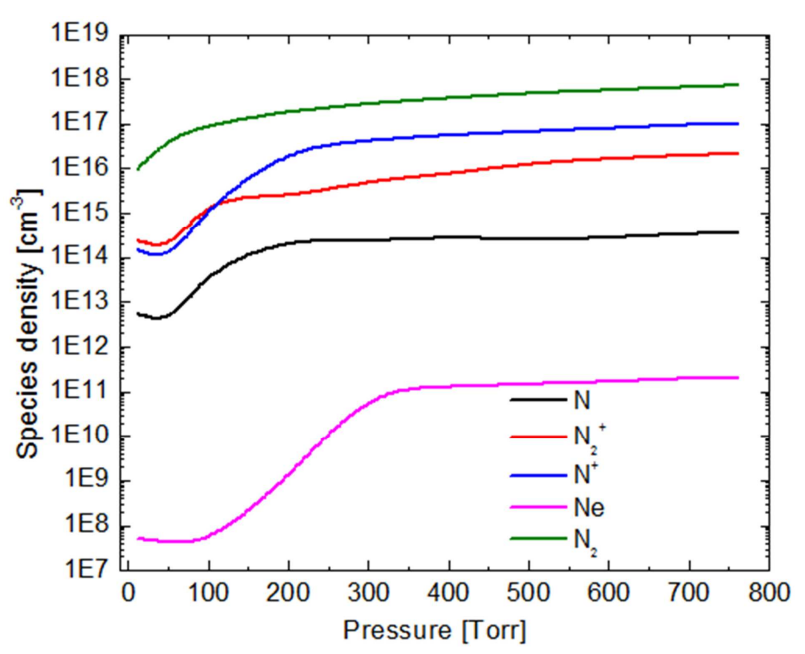

Figure 2. Pressure dependent species densities in nitrogen plasma: $T g=10 \mathrm{kK}, \mathrm{Te}=4.0 \mathrm{eV}$.

The pressure is varied from 1 to 760 Torr. Within the pressure ranging from 1 to 350 Torr, as the pressure is increased the kinetic energy of the electron also increases and the electron-neutral collision frequency increases and hence more ionizations are taking place. Because within this pressure range the mean free path [36] $\left(\lambda=\frac{1}{n_{B} \sigma}\right.$ where $n$ is particle density and $\sigma$ is the collisional cross section [37]) of the electron is larger than those of above 350 Torr. So the electron gets sufficient time to acquire energy from the applied electric field due to their high mobility. As a result the electron-neutral collision is increased with increasing pressure and hence the electron density sharply increases in the pressure ranging from 1 to 350 Torr.

Picking up energy from the electric field the electron transfers it to the heavy plasma constituents through collisions. When the pressure is increased above 350 Torr the electron-neutral collision also increases, but in this case the mean free path of the electron becomes much less than those of pressure below ranging from 1 to 350 Torr. So the electron does not get sufficient time to acquire energy from the applied electric field to make more collisions with different species at a high rate than in the previous case. Eventually the rates of three-body electron recombination reactions balance the rate of ionization, and steady state of the electron density is reached. So the electron density becomes saturated above pressure 350 Torr which is shown in figure-2. The species density of $N, N_{2}, N^{+}, N_{2}^{+}$increase in the pressure ranging from 1 to 200 Torr. Because within this pressure range as the pressure is increased the collision frequency of the species increases and the mean free path of the species are large. As a result in this pressure range the species densities increases sharply. As the pressure is increased above 200 Torr the collision frequency [37] of the species increases but the mean free path becomes more less than the above pressure ranging from 1 to 200 Torr. Hence we can conclude that at high pressure although the collision between different species increases but their production and destruction rates also becomes nearly equal. So at high pressure above 200 Torr the species density becomes approximately saturated.

\subsubsection{Temperature Dependent}

Figure-3: shows the temperature dependent species density of nitrogen plasma.

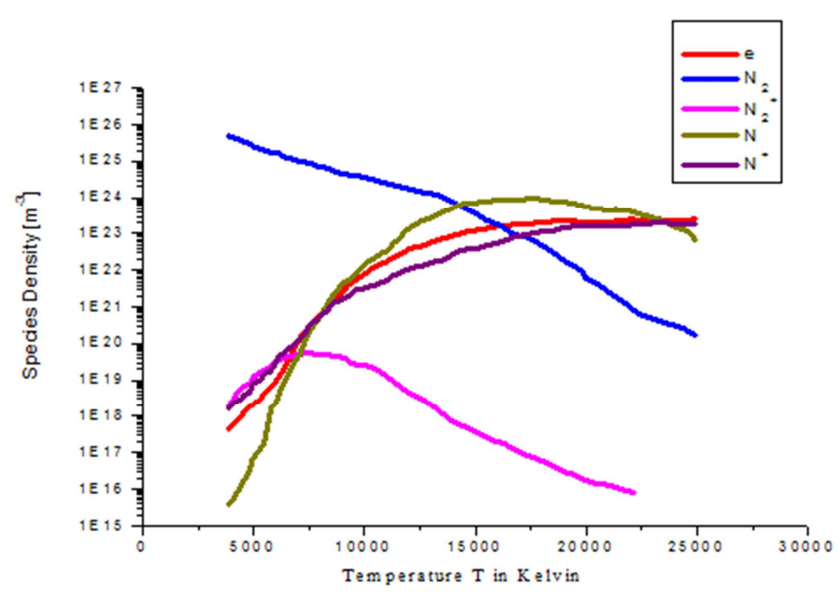

Figure 3. Temperature dependent species densities in nitrogen plasma.

The temperature is varied from 2000 to 25000 Kelvin. Below temperature 2000 Kelvin the density of $N_{2}$ is high. As the temperature is increased the density of $N_{2}$ decreases and the density of electron increases. This is because with increasing the temperature from 2000 to 15000 Kelvin the kinetic energy of the electron increases and electron-neutral collision frequency also increases and hence more ionization can taking place. The mean free path of the electron is higher than that of above temperature 15000 Kelvin and the electron gets sufficient time to acquire energy from the applied electric field. So the density of electron increases due to ionization and the density of $N_{2}$ decreases monotonically. The density of $N$ increases rapidly as the temperature is increased from 3000 Kelvin to 15000 Kelvin and it reaches at a maximum value of approximately $1 \times 10^{24} \mathrm{~m}^{-3}$. Because with increasing the temperature in this range the collision frequency of the species of $N$ also increases. Hence the production rate of $N$ is greater than the destruction rate of it. The density of $\mathrm{N}_{2}^{+}$increases with increasing the temperature ranging from 2000 to 5500 Kelvin. After this temperature range its density decreases rapidly due to increasing their recombination rate with increasing temperature. Above temperature 15000 Kelvin the density of 
electron becomes saturated. Because above this temperature range electron-neutral collision frequency becomes sufficiently high but mean free path becomes much less than that of the temperature ranging from 2000 to 15000 Kelvin and the electron does not get sufficient time to acquire energy from the applied electric field. Hence the destruction and production rates become approximately equal. So the density of electron becomes saturated above temperature 15000 Kelvin. The species density of $N^{+}$becomes saturated above temperature 20000 Kelvin. Because above this temperature although the collision frequency increases but the mean free path is much less than that of below temperature ranging from 2000 to 20000 Kelvin and the destruction and production rates become equal. The density of $N$ starts to decreases as the temperature is increased above 20000 Kelvin. Because above this temperature the destruction rate of $N$ is higher than the production of it. The density of $N_{2}^{+}$starts to decrease as the Temperature is increased above 8000 Kelvin. Because above this temperature the recombination rate of $\mathrm{N}_{2}^{+}$increases and the destruction rate is higher than the production of it.

\subsubsection{Time Dependent}

We have calculated the species density as a function of time ranging from 0 to $19 \mathrm{nS}$.

Figure-4 shows the Time dependent species densities in nitrogen plasma at $T_{g}=10 \mathrm{k}$ kelvin and $p=760$ Torr

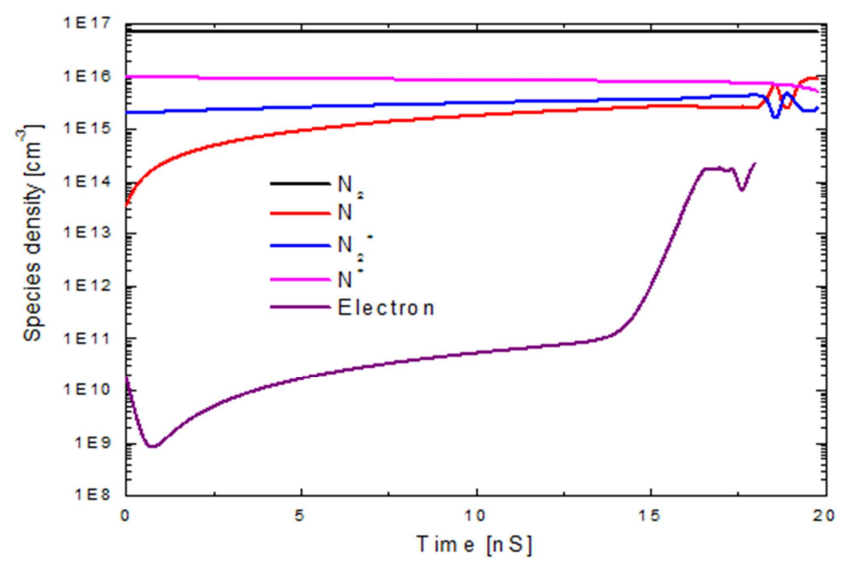

Figure 4. Time dependent species densities in nitrogen plasma: $\mathrm{Tg}=10 \mathrm{kK}, \mathrm{Te}=4.0 \mathrm{eV}, \mathrm{p}=760 \mathrm{Torr}$.

At initial time $t=0 \mathrm{nS}$ the neutral species density of molecular nitrogen is high because the collision is not taking place at this time. At this time the electron does not get sufficient time to acquire energy from the applied electric field. Hence at that time the electron-neutral collision frequency decreases and the electron-ion recombination rate is increased. So the density of electron decreases linearly. After the time above at $t=1 \mathrm{nS}$ the electron density increases because with increasing time the kinetic energy of the species is increased. So the collision frequency also increases and more ionization can take place. So the electron density increases with time. The other species density of nitrogen $N_{2}, N^{+}$and $N_{2}^{+}$are almost constant with time due to their production and destruction rates being equal below time $t=18 \mathrm{nS}$. Above time ranging from nearly 14.5 to $17 \mathrm{nS}$ the electron density increases linearly. Because within this range of time the electron-neutral collision frequency rapidly increase and the electron acquires sufficient energy from the applied electric field and more ionizations can taking place. Hence the production rate of electron is higher than the destruction rate of it. Within time ranging from 18 to $19 \mathrm{nS}$ fluctuations of the species density of $N_{2}^{+}$and $N$ are occurred that means these species density abruptly increase and decrease with time. Because the production and destruction rate of these species change abruptly with increasing the time due to ionization-recombination. At time nearly $17 \mathrm{nS}$ the electron density is fluctuated. Because at this time the recombination and ionization rates of electron with different species increases and decreases rapidly. Hence the production and destruction rates of electron increases abruptly.

\section{Conclusions}

Kinetic model has developed to simulate the transient behavior on the plasma composition properties of nitrogen plasma at atmospheric pressure. This model allows using the simulation of composition, temperature and pressure variations. The kinetic model can be applied for plasmas of any collisional. Results from the model show that, although initially the nitrogen plasma density was low but sharply increases with increasing pressure. The electron density increases from pressure 1 to 350 Torr and after that the density becomes saturated. The densities of nitrogen species increase from pressure 1 to 200 Torr and above that they become saturated. So the production rate of nitrogen plasma from pressure 1 torr to 200 Torr is excellent. But after then the production rate of nitrogen plasma increases slowly. The species density as a function of temperature from 2000 to 25000 Kelvin has investigated and it shows that with increasing temperature the species densities of $N, N^{+}$, and electron density increase but the density of $N_{2}$ decreases with increasing temperature. The density of $\mathrm{N}_{2}^{+}$increases below temperature 7500 Kelvin and above that its density decreases. The production and destruction rates of nitrogen plasma as a function of time was successfully calculated at the time ranging from 0 to $19 \mathrm{nS}$. This result shows the electron density increases with increasing time but the density of $N_{2}, N^{+}$and $N_{2}^{+}$are almost constant. The density of $N$ increases with time from 1 to $5 \mathrm{nS}$ then it becomes saturated.

\section{References}

[1] https://farside.ph.utexas.edu/teaching/plasma/lectures1/node9. html

[2] https://en.wikipedia.org/wiki/Electron_temperature

[3] https://en.wikipedia.org/wiki/Plasma_(physics)

[4] http://kids.britannica.com/comptons/article-299491/energy

[5] https://en.wikipedia.org/wiki/Plasma_parameters

[6] Bell, A. T., in Techniques and Applications of Plasma Chemistry, eds. John R. Hollahan and Alexis T. Bell, p. 1. New York: John Wiley \& Sons, 1974. 
[7] http://www.plasmatreat.com/plasma-technology/plasmaprocesses.html

[8] https://books.google.com.bd/books?id=ZS1JRAeL95sC\&pg=P A42\&lpg=PA42\&dq=Intermediate + pressure + or + medium + pre ssure + plasma\&source $=$ bl\&ots $=j q E A G D j B 8 q \&$ sig $=P-$ hmmAGqxgxzI5Bota RRtiyx0Q\&hl=en\&sa $=$ X\&redir esc $=y$ \#v=onepage\&q=Intermediate $\% 20$ pressure $\% 20$ or\%20medium $\% 20$ pressure $\% 20$ plasma $\& \mathrm{f}=$ false

[9] http://link.springer.com/article/10.1007/BF00903946\#page-2

[10] https://en.wikipedia.org/wiki/Atmospheric-pressure_plasma

[11] https://en.wikipedia.org/wiki/Continuity_equation

[12] http://www.chemguide.co.uk/physical/basicrates/arrhenius.ht $\mathrm{ml}$

[13] https://en.wikipedia.org/wiki/Reaction_rate_constant

[14] Concepts of modern physics, Beiser, $\left(5^{\text {th }}\right.$ edition $)$ Tata McGraw-Hill(1997)

[15] http://csep10.phys.utk.edu/astr162/lect/light/ionization.html

[16] https://en.wikipedia.org/wiki/Electron_ionization

[17] T. Vijayan and Jagadish G. Patil, IEEE Transactions on plasma science,vol. 39, No. 11, November 2011.

[18] B. Chapman, Glow discharge processes, A Wiley-Interscience publication, John Wiley \& Sons, New York.

[19] https://en.wikipedia.org/wiki/Plasma_recombination

[20] E. E. Ferguson, F. C. Fehsenfeld, and A. L. Schmeltekopf, Phys. Rev. A, Vol. 138, 381 - 385, 1965.

[21] Flannery, M. R. (1980), Charge transfer in three-body ion-ion recombination at low gas densities. Int. J. Quantum Chem., 18: 477-482. doi:10.1002/qua.560180850

[22] Physics Department, University of Pittsburgh, Pittsburgh, Pennsylvania 15213 Received December 17, 1968

[23] www.divaportal.org/smash/get/diva2:515728/FULLTEXT01.pdf

[24] Plasma Kinetics in Atmospheric Gases, MCaitelli C.M. Ferreira B. F Gordiets A. I. Osipov.

[25] N. L. Aleksandrov Usp. Fiz. Nauk 154, 177-206 (February 1988)

[26] M. A. Lieberman, J. Appl. Phys. 65, 4186 (1989).

[27] E. Kawamura, V. Vahedi, M.A. Lieberman and C. K. Birdsall, Plasma Sources Sci. Technol. 8, R45 (1999).

[28] F. A. Haas and N. St J Braithwaite, Plasma Sources Sci. Technol. 9, 77 (2000).
[29] E. Gogolides and H. H. Sawin, J. Appl. Phys. 72, Vol. 9, 3971 - 3987, 1992.

[30] R. Veerasingam, R. B. Campbell and R. T. McGrath, Plasma Sources Sci. Technol. 6, 15 169, 1997.

[31] X. M. Zhu and M. G. Kong, J. Appl. Phys. 97, 083301, 2005.

[32] T. J. Sommerer and M. J. Kushner J. Appl. Phys. Vol. 71, No. 4, 15 February 1992.

[33] Yasunori Tanaka, TMichishitia and Y Uesugi,Plasma Sources Sc. Technol.14(2005) 137,doi:10.1088/0963-0252/14/1/016.

[34] Ph Teulet, J J Gonzalez, A Mercado-Cabrera, Y Cressault and A Gleizes, J. Phys. D: Appl. Phys. 42 (2009) 175201 (15pp)

[35] R. J. Shul S. J. Pearton (Eds) Hand book of plasma processing techniques

[36] http://hyperphysics.phyastr.gsu.edu/hbase/kinetic/menfre.html

[37] Plasma Physics and Engineering, Alexander Fridman and Lawrence A. Kennedy, Published in 2004 by Taylor \& Francis 29 West 35th Street New York, NY 10001-2299.

\section{Biography}

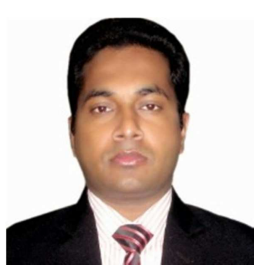

Md. Ziaur Rahman has received his B.Sc.(Hon's) \& Master's degree in Applied Physics \& Electronic Engineering from Rajshahi University, Rajshahi Bangladesh. Currently he is a Lecturer Dept. of Civil Engineering, Dhaka International University, Dhaka, Bangladesh. His current research interest is in the area of Wireless Communication, Digital Signal Processing, Plasma Physics and Renewable Energy.

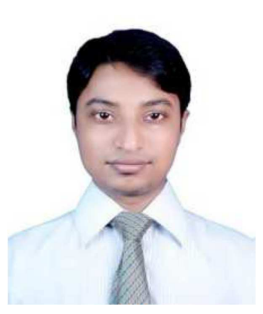

Mohammed Mynuddin has received his B.Sc. degree with Engineering in Electrical and Electronic Engineering (EEE) from Chittagong University of Engineering \& Technology (CUET), Chittagong-4349, and Bangladesh, in 2011. He is a Lecturer dept. of EEE at Daffodil International University, Dhaka, Bangladesh. Currently he is doing his M.Sc. in Electrical \& Electronic Engineering at Georgia Southern University, Georgia, USA. He is a member of the Institution of Engineers, Bangladesh (IEB) His membership ID is M-32776. His current research interest is in Control System, Digital Signal Processing, Industrial Automation, Power System Analysis and Electrical Machine, Renewable Energy. 